\title{
FRACTAL AGGREGATES EVOLUTION DURING FLOCCULATION
}

\author{
R. B. Moruzzi ${ }^{1 *}$, A. L. de Oliveira ${ }^{2}$ and T. de Almeida ${ }^{2}$ \\ ${ }^{1}$ Instituto de Geociências e Ciências Exatas, Univ. Estadual Paulista (UNESP) - Avenida 24-A, nº 1515, C. P. 178, CEP 13506-900, \\ Jardim Bela Vista, Rio Claro, São Paulo, Brazil. \\ ${ }^{2}$ Programa de Pós-graduação em Engenharia Civil e Ambiental, Univ. Estadual Paulista (UNESP), Av. 24-A, 1515, \\ Jardim Bela Vista, Rio Claro, 13506-900. São Paulo, Brazil.
}

(Submitted: May 5, 2017 ; Revised: December 2, 2017 ; Accepted: December 4, 2017)

\begin{abstract}
A temporal analysis of fractal aggregates during water flocculation is presented. Water was prepared using kaolin solution and a non-intrusive image-based analysis method was used for two-dimensional fractal dimension assessment $\left(d_{F}\right)$. The $d_{F}$ values were determined for aggregates larger than $270 \mu \mathrm{m}$ from 100 images taken in each combination of velocity gradients $\left(G_{f}\right)$ and flocculation times $\left(T_{f}\right)$. The $G_{f}$ values of $20,30,40,50$ and $60 \mathrm{~s}^{-1}$ and $T_{f}$ values of 2 to 180 minutes were studied, with a $\Delta T_{f}$ of 1 minute for $2 \leq T_{f} \leq 10$ minutes and a $\Delta T_{f}$ of 5 minutes for $10 \leq T_{f} \leq 180$ minutes. Results showed that $d_{F}$ tends toward stability from a $T_{f}$ of 40 minutes, with distinct levels in each $G_{f}$. Further, $d_{F}$ values decrease from 1.80 to 1.35 for $G_{f}$ from 20 to $60 \mathrm{~s}^{-1}$.

Keywords: Flocculation; Fractal aggregates; Temporal evolution.
\end{abstract}

\section{INTRODUCTION}

The flocculation project must contain information about the geometry and arrangement of the tanks, which determine the residence time in hydraulic flocculation chambers, as well as the mixing conditions provided by the hydraulic or mechanical equipment. Thus, the pair of values given by the medium gradient velocity and by the flocculation time determines the distribution size and shape of the effluent aggregates.

In this context, the project improvement and flocculator operation requires the understanding of the mechanisms involved during flocculation. The classic representation of the phenomenon of flocculation involves two discrete steps: transport and adhesion.

The transport of particles arises from three mechanisms that act simultaneously, namely, perikinetic, defined as the movement erratically driven by thermal energy; orthokinetic, arising from the mixture imposed on the system by means of hydraulic or mechanical agitation and measured through the velocity gradient; and differential sedimentation, arising from the terminal velocities of different particles with specific masses and sizes, as originally proposed by Smoluchowski (1917) and mentioned by Thomas et al. (1999), and Camp and Stein (1943).

The adherence, in turn, depends on a series of surface phenomena and approximation forces, such as electrostatic repulsion and attraction of van der Waals forces, described by Deryaguin and Landau (1941) and by Verwey and Overbeek (1948). Further, the adherence varies according to the particle's nature and the destabilization method employed to overcome the repulsive energy barrier that dominates the interparticle interaction, keeping them stable. Thus, the success of flocculation depends mainly on the rate of particle collisions and attachment.

The model proposed by Smoluchowski (1917) is a milestone in flocculation research, as mentioned by Watanabe (2017). The basic equation developed for discrete particles of sizes $i, j$ and $k$ is given by Equation 1 .

$$
\frac{d n_{k}}{d t}=\frac{1}{2} \sum_{i+j=k} H(i, j) n_{i} n_{j}-\sum_{i, j=1}^{\infty} H(i, j, k) n_{i, j} n_{k}
$$

\footnotetext{
* Corresponding author: Phone: +55 19 3526-9339. E-mail: rmoruzzi@rc.unesp.br. ORCID: https://orcid.org/0000-0002-1573-3747
} 
where $i$ represents discrete sized particles $i ; j$ represents discrete sized particles $j ; k$ represents particles sized $k$; $H(i, j)$ is the collision rate between the particles $i$ and $j$ $\left(\mathrm{L}^{3} \mathrm{t}^{-1}\right) ; H(i, k)$ is the collision rate between the particles $i$ and $k\left(\mathrm{~L}^{3} \mathrm{t}^{-1}\right) ; n_{i}$ is the number of particles of size $i$ per volume unit $\left(\mathrm{L}^{-3}\right) ; n$ is the number of particles of size $j$ per volume unit $\left(\mathrm{L}^{-3}\right)$; and $d n_{k} / d t$ is the variation of the number of particles $k$ per time.

The first term on the right hand side describes the increase in the number of particles of size $k$ by flocculation of two particles of sizes $i$ and $j$, whose sum results in a volume equal to the volume of a particle of size $k$. The multiplicative factor ensures that the same shock is not counted twice, either by particles $i$ in $j$ and vice versa. The second term on the right describes the disappearance of particles of size $k$ due to their aggregation with particles of sizes $i$ or $j$.

Upon proposing Equation 1, Smoluchowski made a number of simplifications, namely, (i) the efficiency of collision is unique for all particles; ii) the flow is laminar; (iii) the particles are monodisperse; iv) there is no rupture of flocs previously formed; (v) all particles have a spherical shape and remain spherical after the collision; and vi) collisions involve only two particles. Based on these assumptions, analytical expressions were developed by Smoluchowski to describe the frequency of collisions between particles for perikinetic and orthokinetic flocculation, given by equations 2 and 3 , respectively.

$$
\begin{aligned}
& H(i, j)=\left(\frac{2 \cdot k \cdot T}{3 \mu}\right) \frac{d_{j}}{d_{i}+1}\left(d_{i}+d_{j}\right) \\
& H(i, j)=\frac{1}{6} \frac{d v}{d y}\left(d_{i}+d_{j}\right)^{3}
\end{aligned}
$$

where $k$ is the Boltzmann constant $\left(\mathrm{M} \mathrm{L}^{2} \mathrm{t}^{-2} \mathrm{~T}^{-1}\right) ; T$ is the temperature $(\mathrm{K}) ; \mu$ is the absolute viscosity $\left(\mathrm{ML}^{-1} \mathrm{t}^{-1}\right) ; d_{i, j}$ is the diameter of particles of size $i$ or $j$ (L); $i$ represents discrete particles of size $i ; j$ represents discrete particles of size $j ; \mathrm{H}(i, j)$ is the rate of collision between particles $i$ and $j\left(\mathrm{~L}^{3} \mathrm{t}^{-1}\right)$; and $d v / d y$ is the velocity gradient in the fluid $\left(\mathrm{t}^{-1}\right)$.

One of the assumptions of the classic proposal of Smoluchowski (1917), as cited above, provides that all particles are spherical and remain spherical after aggregation. Further, the kinetic approach presented by Argaman and Kaufman (1970) showed that the dynamic steady state is reached when the effects of aggregation and breakage are acting equally upon aggregates.

Different approaches comprise the irregular and disorderly nature of particles in which the floc porosity is considered, as presented by Parker et al. (1972) and Tambo and Hozumi (1979). More recently, the theory of fractals was incorporated into the analysis of porosity and shape of particles, as shown in Thomas et al. (1999), Li and Logan (1997), Kim and Kramer (2006), Li et al. (2007), Gregory (2009) and Wu and He (2010), among others.

Li and Ganczarczyk (1989) present Equation 4 to describe the relationship between porosity and the size of the fractal.

$C=1-S \cdot R^{d_{F}-3}$

where $C$ is the aggregate porosity (-); $S$ is a specific constant; $R$ is the radius of the particle (aggregate) (L); and $d_{F}$ is the dimension of fractal, expressed as a diameter (L).

The value $d_{F}$ of Equation 4 represents the threedimensional fractal dimension and it varies from 1 to 3 , where if $d_{F}=3$, the particle is perfectly spherical (3D), when the density is constant and independent of particle size. For $d_{F}=1$, the density has a high degree of dependence on particle size, and the particle has a completely open structure. For the Smoluchowski equation there is an implicit assumption that the threedimensional fractal dimension is 3, i.e., a perfect sphere (Thomas et al., 1999).

Equation 5, proposed by $\mathrm{Li}$ and Logan (1997), represents the relationship between the collision frequency $(H)$ and the size of the fractal for flocculation in a turbulent field.

$H \approx G_{f}^{\left(1-d_{F} / 3\right)}$

where $H$ is the collision frequency $\left(\mathrm{t}^{-1}\right) ; G_{f}$ is the medium velocity gradient of flocculation $\left(\mathrm{t}^{-1}\right)$; and $d_{F}$ is the three-dimensional fractal dimension (-).

Therefore, a steady-state is supposed during flocculation since aggregation and breakage rates result in aggregate size stable over time (Jarvis et al., 2005). Nevertheless, aggregates with the same size may have different structures (i.e., fractal dimensions) as a result of different arrangements of primary particles within aggregates during flocculation (Vahedi and Gorczyca, 2012; Moruzzi et al., 2017). Further, properties such as density and the velocity of sedimentation are naturally not integrated to the size, but they exhibit relations with the dimension of fractal, as mentioned by Gregory (2009).

Hence, fractal structure analysis applied to aggregates is currently a new tool to approach complex flocculation mechanisms, as shown by Zhong et al. (2011), Yang et al (2013), Ringl and M. Urbassek (2013), Wei (2015), Abdellatif et al. (2016), Moruzzi et al (2017) and Wilkinson, et al. (2017), among others. The morphological aspects of aggregates, mainly in terms of fractal dimension, have been 
studied for assessment of flocculation performance and for enhance mathematic modeling, which was first concerned with Euclidean geometry.

In this context, the morphological evolution of aggregates during the flocculation is as important as the evolution of size. This is essential in order to broaden the understanding about the effect of shear rate and time on the dimension of fractal aggregates during flocculation.

This paper assessed the aggregate evolution of the two-dimensional fractal dimension during flocculation. Further, the assessment of the aggregate morphological stability from the steady-state size was also investigated.

\section{METHODOLOGY}

Synthetic water was used to better control assay conditions and reduce interferences caused by natural water variations, as also adopted by several authors (Chackraborti et al., 2003; Gregory, 2009; Zhong et al., 2011; He et al., 2012; Moruzzi et al., 2017, among others). The solution of kaolin was prepared according to the procedure described in Yukselen and Gregory (2004). Forty grams of kaolin was introduced into 1 L of deionized water, and the mixture was performed for two hours with a medium velocity gradient of 1000 $\mathrm{s}^{-1}$. At the end of the agitation, the solution was left to stand for 12 hours, and the supernatant, corresponding to approximately $800 \mathrm{~mL}$, was removed to form the stock solution. The turbidity of the mother suspension was $5000 \pm 200$ NTU. For the study samples, $10 \mathrm{~mL}$ of the solution were diluted in $2 \mathrm{~L}$ of water to give a turbidity of $25 \pm 2$ NTU.

The batch assays were performed using a Jar test. Previous experiments to obtain the diagrams of coagulation/flocculation were carried out, with the coagulation $\mathrm{pH}$ varying from 4 to 9 and the dosage of coagulant (hydrated aluminum sulfate $\mathrm{Al}_{2}\left(\mathrm{SO}_{4}\right)_{3} .16 \pm$ $2 \mathrm{H}_{2} \mathrm{O}$ ) varying from 0.25 to $4.75 \mathrm{mgAl}^{+3} . \mathrm{L}^{-1}$.

For flocculation assays, the best coagulation condition obtained in the previous step was used. The values of $G_{f}$ varied from 20 to $60 \mathrm{~s}^{-1}\left(\Delta G_{f}=10 \mathrm{~s}^{-1}\right)$ and values of $T_{f}$ varied from 2 to 180 minutes, with a $\Delta T_{f}$ of 1 minute from $2 \leq T_{f} \leq 10$ minutes and with a $\Delta T_{f}$ of 5 minutes from $10 \leq T_{f} \leq 180$ minutes. Aggregate growth was monitored with an image analysis apparatus, following the procedure described in Moruzzi and Reali (2010) and adapted by Moruzzi et al. (2016). For these assays, the images were obtained at $10 \mathrm{~Hz}$ in the final 10 seconds of each $T_{f}$. Thus, 215 samples were obtained, 100 images each, for each value of $G_{f}$ investigated, resulting in 21,500 analyzed images with an average of 164.7 flocs per image; in total, 3,541,050 aggregates were assessed. For the two-dimensional fractal dimension only aggregates larger than 270 $\mu \mathrm{m}$ were considered, following the recommendation of Chakraborti et al. (2003). In Figure 1, a simplified scheme of the image apparatus and an example of an aggregate image captured in situ during a typical essay are shown.

The reason why Alum was used as coagulant, as well as why velocity gradients and flocculation time were chosen, is due to the fact that they are common and within the normal range used in the treatment process. Also, several authors since the classical studies of Argaman and Kaufman (1970) and Bratby (1981) to more recent works like Chackraborti et al. (2003), Li et al. (2007), Zhong et al. (2011), Moruzzi et al. (2017) and Yu et al. (2015) used Alum as the primary coagulant, making comparison possible.

Statistical analyses were performed using descriptive statistics, 95\% confidence interval, quantile regression, hypothesis testing and a test for comparison of Tukey's means, aiming to obtain the minimum significant difference. The sampling error on average was determined to a confidence of $99 \%$ ( of standard normal distribution of 2.329), considering an infinite population of aggregates.

Digital images were analyzed with the software Image-pro Plus $\AA$, coupled to a specific computational routine for semi-automation. The error resulting from the treatment of a digital image was estimated by assessing the relationship between the areas produced by the outline of the object and those produced by
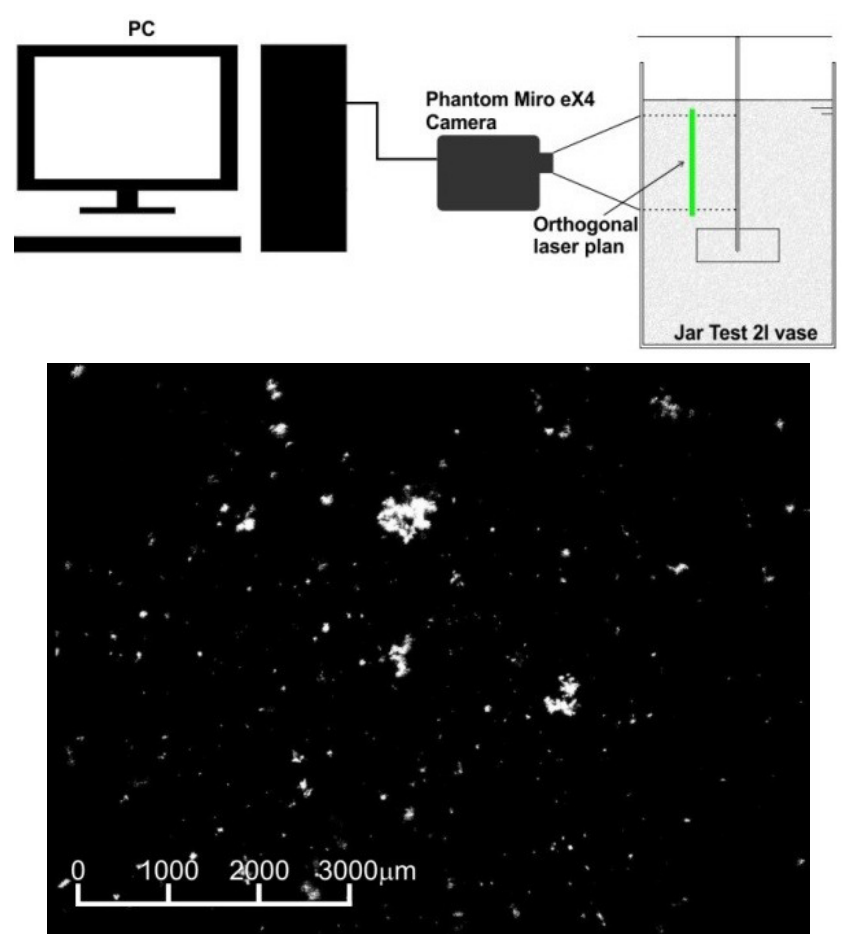

Figure 1. Simplified scheme of the image apparatus and an example of an aggregate image captured in situ during a typical assay. 


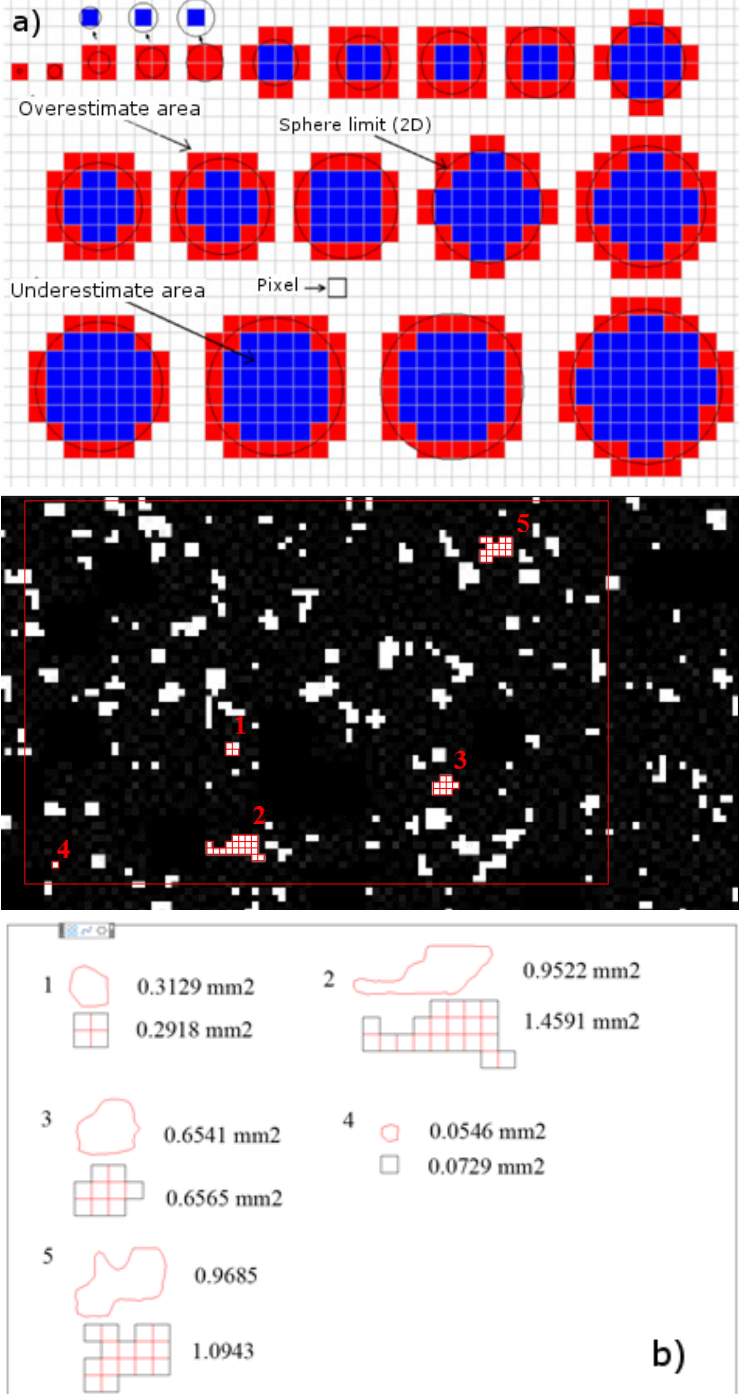

Figure 2. Illustrative procedure used to estimate the error inherent to the digital picture treatment considering the elementary unit as a reference (pixel) on the display grid. Error is defined by the ratio between the image digital resolution and the visual field for geometric regular (a) and irregular (b) projections.

the elementary unit of a digital image (pixel) in two configurations, i.e., over and underestimation (Figure 2). In this case, the equivalent sphere of the same projected area was used, as described in Santos et al. (2004).

Two-dimensional fractal dimension was addressed using equation 6 and the results were plotted in a lnln plot, Equation 7, presented by Bushell and Amal (1998).

$A \approx D^{d_{F}}$

$\ln (A)=d_{F} \cdot \ln (D)$

where $A$ corresponds to the area of the particle projected onto the plane of the image; $D$ corresponds to the equivalent characteristic in area; and $d_{F}$ corresponds to the exponent of the ratio between area and diameter, defined as a diameter of fractal to the area, which is the slope of the plot of $\ln (\mathrm{A})$ versus $\ln (\mathrm{D})$.

\section{RESULTS AND DISCUSSION}

The errors from the digital image treatment are presented in Table 1. The error decreases as the range of particle sizes increases. Results for a collection of irregular shaped aggregates are depicted in Table 2. The error ranged from 0.19 to $19 \%$ referring to the projected area. It is worth mentioning that the error is less apparent along the dimension of fractal, as described by Equation 7 .

Results of descriptive statistics for the twodimensional fractal dimension $\left(d_{F}\right)$ are presented in Table 3. For the set sampled, the $p$-value was 0.002 with a $95 \%$ confidence interval. The frequency distribution of the data obtained can be found in Figure 3 . It is clear that most of aggregates (ca $80 \%$ ) were in the $d_{F}$ range of 1.4 and 1.6. The aggregates present an open structure and the density depends on their size. An open structure may also represent less stress resistance, since it is dependent on primary particle bonds (He et al., 2012).

The evolution of the two-dimensional fractal dimension $\left(d_{F}\right)$ aggregates for different values of $G_{f}$ can be observed in Figure 4. Each $d_{F}$ value plotted in Figure 4 is the result of 100 image analyses (ca. 16,500 aggregates per $d_{F}$ ), as exemplified on the right side of the same figure.

Table 1. Estimated errors in the image treatment (binarization) for each range of floc sizes in regular geometric figures (as shown in Figure 2a).

\begin{tabular}{|c|c|c|c|c|c|}
\hline \multicolumn{2}{|c|}{ Range } & \multirow{2}{*}{$\begin{array}{c}D_{\text {medium }} \\
\text { of the floc }\end{array}$} & \multirow{3}{*}{$\begin{array}{c}\text { Real } \\
\text { Area* } \\
\left(\mathrm{mm}^{2}\right)\end{array}$} & \multirow{2}{*}{\multicolumn{2}{|c|}{ Range of Error $(\%)$}} \\
\hline $\mathbf{D}_{\min }$ & $\mathbf{D}_{\max }$ & & & & \\
\hline \multicolumn{3}{|c|}{$(\mathrm{mm})$} & & Underest. & Overest. \\
\hline 0.625 & 0.750 & 0.6875 & 0.371 & $11 \%$ & $54 \%$ \\
\hline 0.875 & 1.000 & 0.9375 & 0.690 & $35 \%$ & $30 \%$ \\
\hline 1.000 & 1.125 & 1.0625 & 0.887 & $43 \%$ & $15 \%$ \\
\hline 1.250 & 1.375 & 1.3125 & 1.353 & $20 \%$ & $31 \%$ \\
\hline 1.500 & 1.625 & 1.5625 & 1.917 & $22 \%$ & $10 \%$ \\
\hline 1.750 & 1.875 & 1.8125 & 2.580 & $18 \%$ & $21 \%$ \\
\hline 2.000 & 2.125 & 2.0625 & 3.341 & $16 \%$ & $14 \%$ \\
\hline 2.250 & 2.375 & 2.3125 & 4.200 & $13 \%$ & $15 \%$ \\
\hline
\end{tabular}

Table 2. Estimated errors in the image treatment (binarization) for each range of floc sizes in irregular geometric figures (as shown in Figure 2b).

\begin{tabular}{|c|c|c|c|}
\hline \multirow{2}{*}{ Aggregate } & Area Pixels* & Real Area* & \multirow{2}{*}{ Error (\%) } \\
\hline & \multicolumn{2}{|c|}{$\left(\mathrm{mm}^{2}\right)$} & \\
\hline 1 & 0.29 & 0.31 & 3.54 \\
\hline 2 & 1.45 & 0.95 & 19.21 \\
\hline 3 & 0.65 & 0.65 & 0.19 \\
\hline 4 & 0.07 & 0.05 & 13.49 \\
\hline 5 & 1.09 & 0.96 & 5.92 \\
\hline
\end{tabular}


Table 3. Descriptive statistics for the fractal sizes obtained in the flocculation tests for values of $G$ ranging from 20 to $60 \mathrm{~s}^{-1}$ with flocculation times of 2 to 180 minutes.

\begin{tabular}{lccccc}
\hline & $\mathbf{2 0}$ & $\mathbf{3 0}$ & $\mathbf{4 0}$ & $\mathbf{5 0}$ & $\mathbf{6 0}$ \\
\hline Mean & 1.58 & 1.36 & 1.37 & 1.46 & 1.36 \\
Standard error & 0.07 & 0.03 & 0.03 & 0.04 & 0.02 \\
Median & 1.73 & 1.39 & 1.38 & 1.48 & 1.35 \\
Mode & 1.75 & 1.42 & 1.24 & 1.58 & 1.44 \\
Standard deviation & 0.23 & 0.10 & 0.10 & 0.13 & 0.06 \\
Interval & 0.60 & 0.34 & 0.28 & 0.32 & 0.17 \\
Minimum & 1.17 & 1.16 & 1.24 & 1.3 & 1.28 \\
Maximum & 1.77 & 1.50 & 1.52 & 1.62 & 1.45 \\
Confidence Interval (95\%) & 0.16 & 0.07 & 0.06 & 0.09 & 0.04 \\
\hline
\end{tabular}

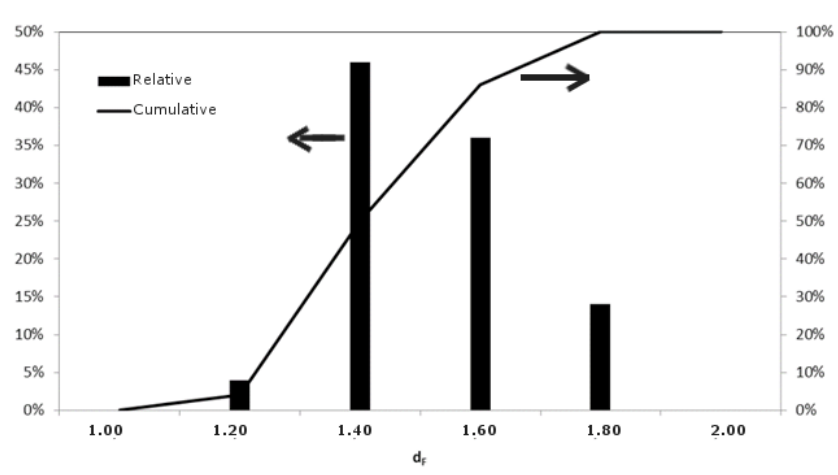

Figure 3. Distribution of relative and cumulative frequency of the two-dimensional fractal dimension $\left(d_{F}\right)$ obtained in the experiments.
All the curves shown in Figure 4 appeared to reach stability of $d_{F}$ values, leading to a steady-state (EE), and also each $G_{f}$ value leads to a different position in the EE. In general, a higher $d_{F}$ (c.a 1.8) was obtained for the lower $G_{f}$ value $\left(20 \mathrm{~s}^{-1}\right)$. This indicates a more regular shape for $G_{f}$ of $20 \mathrm{~s}^{-1}$ for $T_{f}$ higher than 10 min. (Li and Ganczarczyk, 1989). With the increase of $G_{f}$ values, a decrease of the $d_{F}$ values was observed, as also reported by Parker et al. (1972), Tambo and Hozumi (1979), Thomas et al. (1999) and Li and Logan (1997).

Similarly, the overall $d_{F}$ evolution can be divided into two phases, a transitional phase (TP) and a steadystate phase (EE), as also observed by He et al. (2012). For TP, $d_{F}$ values tend to rapidly increase to a peak, indicating that shape is dramatically changed by the aggregation stage. The TP phase seems to occur very rapidly in the first few minutes of flocculation $\left(T_{f} \leq 5 \mathrm{~min}\right.$.), and the greater the $G_{f}$ the shorter the time to reach the peak (Figure 5). Subsequently, $d_{F}$ tends to remain constant $\left(G_{f}\right.$ of $\left.20 \mathrm{~s}^{-1}\right)$ or to decrease to a lower level, from which EE can be observed. In general, the EE occurred in flocculation times lower than 40 minutes $\left(T_{f} \leq 40 \mathrm{~min}\right.$.), as shown in Figure 5. However, it is clear that EE initiates at different $T_{f}$ for the investigated $G_{f}$ values. In addition, the $d_{F}$ value in $\mathrm{EE}$ decreases as the $G_{f}$ increases from 1.80

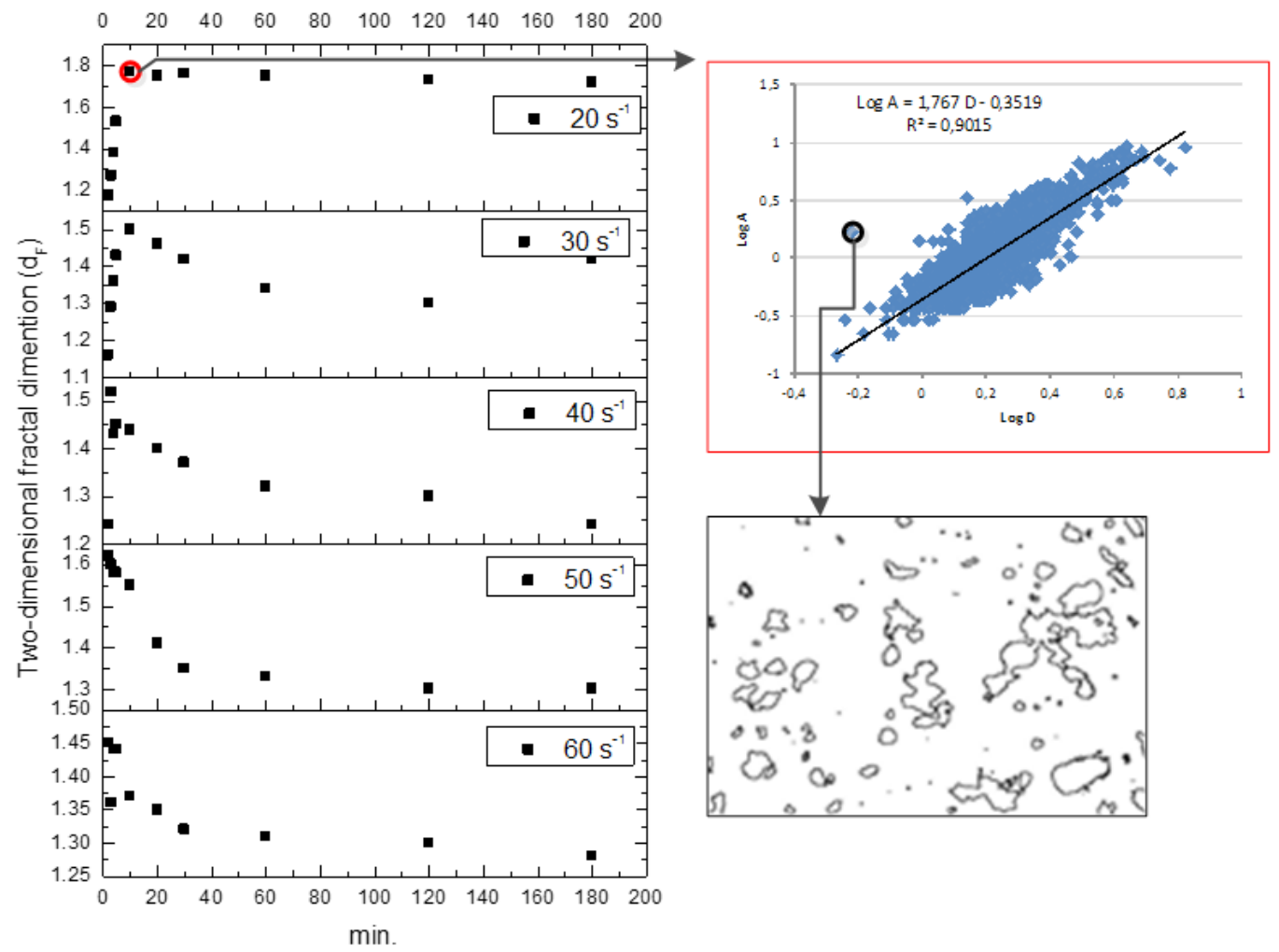

Figure 4. Temporal evolution of the two-dimensional fractal dimension $\left(d_{F}\right)$ for different values of velocity gradients $\left(G_{f}\right)$. Standard deviation and confidence interval for $95 \%$ are presented in Table 1 . On the right side, an example of $d_{F}$ assessment from the projected area for the whole aggregate population and its linearized relationship with the mean diameter of classes (D). 


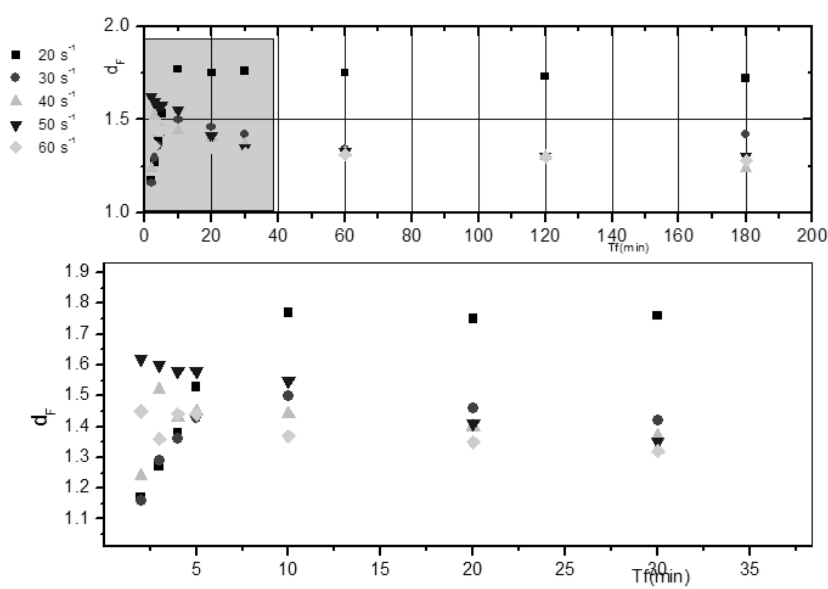

Figure 5. Compilation of the diameter of fractal results $\left(d_{F}\right)$ for different values of applied velocity gradients $\left(G_{f}\right)$. The expansion of the region, defined by the time interval of flocculation $\left(T_{f}\right)$ to values lower than 40 minutes, is as follows.

to 1.35 for values of $G_{f}$ from 20 to $60 \mathrm{~s}^{-1}$, respectively, in accordance with the experiments presented by Chakraborti et al. (2003), where values ranging from 1.83 to 1.51 were observed.

The data quartile distribution for the entire range of $T_{f}\left(2<T_{f} \leq 180\right.$ minutes $)$, as shown in Figure 6, indicates larger oscillations for the $G_{f}$ value of $20 \mathrm{~s}^{-1}$, while the other $G_{f}$ values produced narrower distributions. These are the consequence of the above mentioned behavior, since that for $20 \mathrm{~s}^{-1}$ it took more time to reach the peak and so values varied in a wide range.

Despite the wide range of $d_{F}$ values, especially due to the TP phase, the hypothesis test of the $d_{F}$ temporal variations, for all $G_{f}$ and $T_{f}$ values investigated, led to rejection of the null hypothesis ( $\mathrm{p}$ value $<0.05$ ), indicating that at least two treatments are significantly different from each other.

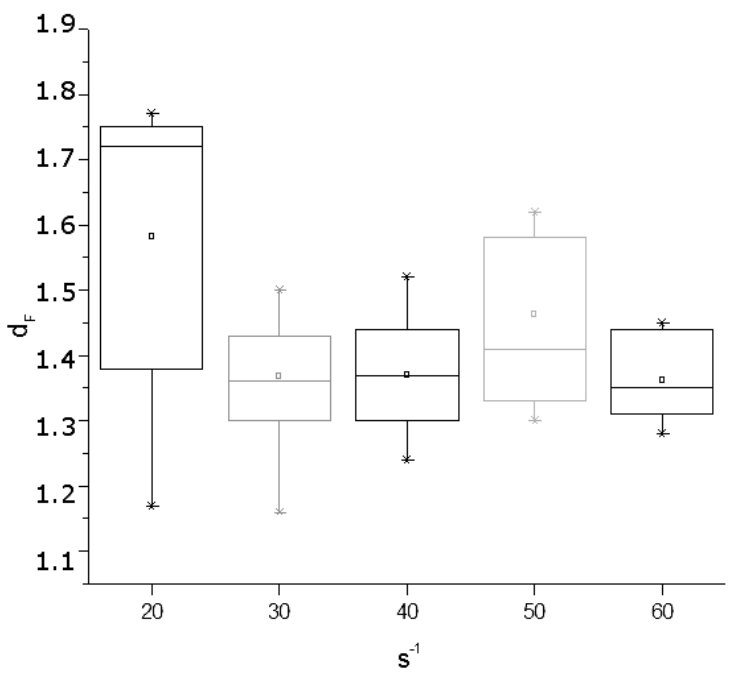

Figure 6. Quartile distribution of the diameter of the fractal $\left(d_{F}\right)$ for different applied values of medium velocity gradients $\left(G_{f}\right)$.
Results presented in Figure 7 show the band of $d_{F}$ values within the $95 \%$ confidence interval. It is clear that the higher $G_{f}$ tend to vary in a narrower range, as a consequence of the shorter TP observed. For $G_{f}$ of 30, 40 and $60 \mathrm{~s}^{-1}, d_{F}$ values varied within a close band, and they differed from the $d_{F}$ values for 20 and $50 \mathrm{~s}^{-1}$. Pairwise comparison presented in Figure 8 shows that $d_{F}$ of $20 \mathrm{~s}^{-1}$ differs from all other, at a statistical level of $95 \%$.

In general, the joint data assessment in terms of diameter of the fractal $\left(d_{F}\right)$ unequivocally leads to the conclusion that the steady-state (EE) occurs for all $G_{f}$ values from a given time $\left(T_{f}\right)$ and that, under the conditions with which the present study was delimited, the EE was observed at flocculation times longer than 40 minutes ( $T_{f} \geq 40 \mathrm{~min}$ ). The observation of the flocculation dynamic stability corroborates observations made by other authors who investigated

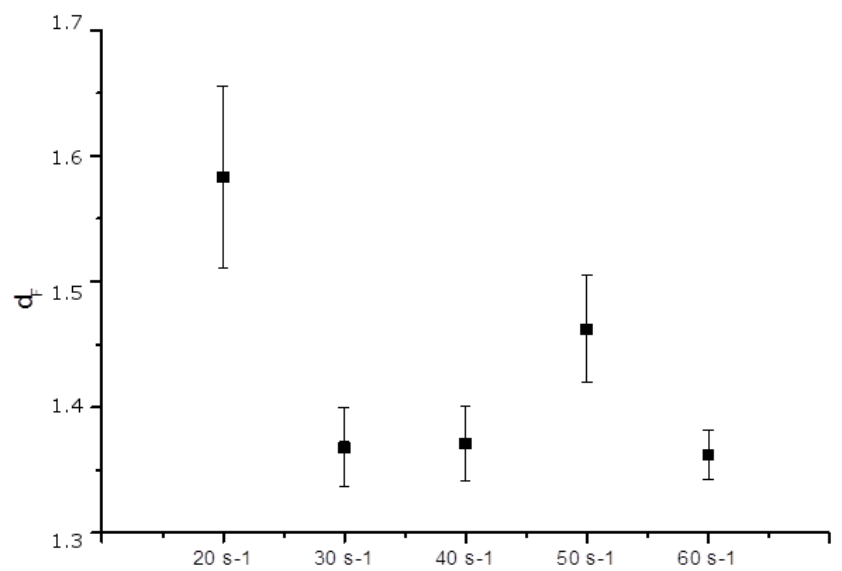

Figure 7. Mean fractal diameter $\left(d_{F}\right)$ for different applied values of medium velocity gradients $\left(G_{f}\right)$ for the $95 \%$ confidence interval.

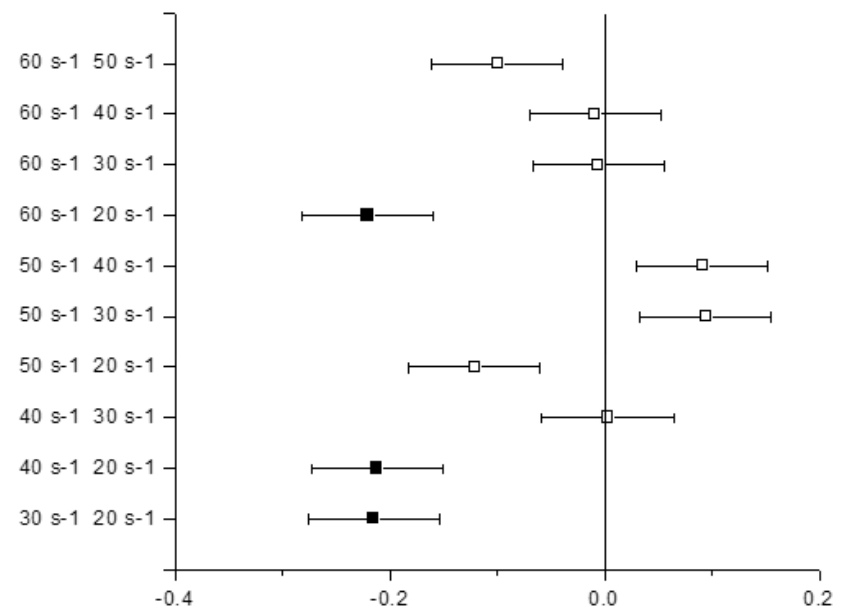

Figure 8. Comparison test of Tukey means to obtain the least significant difference in the fractal diameter $\left(d_{F}\right)$ for $2 \leq T_{f} \leq 180 \mathrm{~min}$ for different applied values of average velocity gradients $\left(G_{f}\right)$, compared two by two. The markers with filling in black are significantly different, while the others cannot be considered significantly different with $95 \%$ significance. 
turbidity measures and the size of aggregates, such as Argaman and Kaufman (1970), Bratby (1981), Haarhoof (1995), Spicer and Pratsinis (1996), Bouyer et al. (2004), and Yang et al. (2013).

Finally, the results obtained reinforce the importance of advancing the analysis of fractal aggregates, and the implications of fractal aggregate analysis for flocculation and subsequent treatment steps.

\section{CONCLUSIONS}

In this article, the temporal analysis of the twodimensional fractal diameter $\left(d_{F}\right)$ during the flocculation of water containing kaolin was investigated through the use of a non-intrusive image technique.

It was observed that the values of $d_{F}$ tend toward stability for $T_{f}$ of 40 minutes, with distinct levels at each $G_{f}$. The value of $d_{F}$ decreased from 1.80 to 1.35 for $G_{f}$ values from 20 to $60 \mathrm{~s}^{-1}$.

In general, thre experimental results of this study confirm the morphological stability, also observed for a given shear rate when monitoring aggregates by size, indicating that fractal evaluation can be a further element for monitoring the performance of the flocculation.

\section{ACKNOWLEDGEMENTS}

Authors are thankful to the São Paulo Research Foundation (Fundação de Amparo à Pesquisa do Estado de São Paulo - FAPESP. Process numbers: 2010/50694-0; 2013/25641-9 and 2017/19195-7 for support of this research. The authors dedicate this work to the memory of Dr Marcelo De Julio who contributed to the advancement of coagulationflocculation research in Brazil.

\section{NOMENCLATURE}

$H(i, j)$ collision rate between the particles $i$ and $j\left(\mathrm{~L}^{3} \mathrm{t}^{-}\right.$ 1)

$H(i, k)$ collision rate between the particles $i$ and $k\left(\mathrm{~L}^{3} \mathrm{t}^{-}\right.$ 1)

$n_{i} \quad$ number of particles of size $i$ per unit volume

$\left(\mathrm{L}^{-3}\right)$

$n_{i} \quad$ number of particles of size $j$ per unit volume

$\left(\mathrm{L}^{-3}\right)$

$d n_{k} / d t \quad$ variation of the number of particles $k$ per time

$k^{k} \quad$ Boltzmann constant $\left(\mathrm{M} \mathrm{L}^{2} \mathrm{t}^{-2} \mathrm{~T}^{-1}\right)$

T temperature $(K)$

$\mu \quad$ absolute viscosity $\left(\mathrm{ML}^{-1} \mathrm{t}^{-1}\right)$

$d_{i j} \quad$ diameter of particles of size $i$ or $j(\mathrm{~L})$

$\mathrm{H}(i, j)$ rate of collision between particles $i j\left(\mathrm{~L}^{3} \mathrm{t}^{-1}\right)$

$d v / d y \quad$ velocity gradient in the fluid $\left(\mathrm{t}^{-1}\right)$
C aggregate porosity (-)

$S \quad$ a specific constant

$R \quad$ radius of the particle (aggregate) (L)

$d_{F} \quad$ fractal dimension, expressed as a diameter (L)

$H \quad$ collision frequency $\left(\mathrm{t}^{-1}\right)$

$G_{f} \quad$ medium velocity gradient of flocculation $\left(\mathrm{t}^{-1}\right)$

$d_{F} \quad$ fractal dimension (-)

$A$ the area of the particle projected onto the plane of the image $\left(\mathrm{L}^{2}\right)$

$D \quad$ the equivalent diameter in area (L)

$d_{F} \quad$ the exponent of the ratio between area and diameter (-), calculated as the slope of the plot of $\ln (\mathrm{A})$ versus $\ln (\mathrm{D})$

\section{REFERENCES}

Abdellatif, M. H., Abdelrasoul, G. N., Salerno, M., Liakos, I., Scarpellini, A., Marras, S. and Diaspro, A. Fractal analysis of inter-particle interaction forces in gold nanoparticle aggregates. Colloids and Surfaces A: Physicochem. Eng. Aspects, 497, 225-232 (2016).

Argaman Y. \& Kaufman W.J. Turbulence and flocculation. J. Sanitary Eng. Div. ASCE 96, 22341 (1970).

Bouyer, D.; Liné, A.; Do-Quang, Z. Experimental Analysis of Floc Size Distribution under Different Hydrodynamics in a Mixing Tank. AIChE Journal, 50(9), 2064-2081(2004).

Bratby, J.R. Interpreting laboratory results for the design of rapid mixing and flocculation systems. Jour. AWWA, 73(6), 318-325 (1981).

Camp, T.R. and Stein, P.C. Velocity gradients and internal work in fluid motion. Journal Boston Society Civil Engineers, 30, 219-237 (1943).

Chakraborti, R.K., Gardner, K.H., Atkinson, J.F., Van Benschoten., J.E. Changes in fractal dimension during aggregation. Water Res, 37, 873-883 (2003).

Deryaguin B. V. and Landau L. D. A theory of the stability of strongly charged lyophobic sols and of the adhesion of strongly charged particles in solutions of electrolytes. Acta Physicochim. USSR14, 633 (1941).

Gregory J. Monitoring particle aggregation processes. Advances in Colloid and Interface Science, 147148, 109-123 (2009).

Haarhoff, J.; Beek, J. C. van; Zyl, H. J. van. Pratical application of the Argaman-Kaufman flocculation model. Department of Civil Engineering, Rand Africaans University. Johannesburg. (1995).

He, W.P., Nan, J., Li, H.Y., Li, S.N. Characteristic analysis on temporal evolution of floc size and structure in low-shear flow. Water Research., 46, 509-520 (2012). http://dx.doi.org/10.1016/j. watres.2011.11.040 
Jarvis, P., Jefferson, B., Gregory, J., Parsons, S.A. A review of floc strength and breakage. Water Res., 39, 3121-3137 (2005). http://dx.doi.org/10.1016/j. watres.2005.05.022

Kim, J.; Kramer, T.A. Improved orthokinetic coagulation model for fractal colloids: Aggregation and breakup. Chemical Engineering Science. 61, 45-53 (2006).

Li D.-H. and Ganczarczyk J. Fractal geometry of particle aggregates generated in water and wastewater treatment processes. Environ. Sci. Technol. 23. 1385-1389 (1989).

Li X.; Logan B. E. Collision frequencies between fractal aggregates and small particles in a turbulently sheared fluid. Environ. Sci. Technol. 31. 1237-1242 (1997).

Li, T.; Zhu, Z.; Wang, D.; Yao, C.; Tang, H. The strength and fractal dimension characteristics of alum-kaolin flocs. Int. J. Miner. Process. 82, 23-29 (2007).

Moruzzi R. B., Oliveira, A. L., De Julio, M., Dutra R. H. A. \& Silva, P. A. G. Floculação: considerações a partir da análise clássica e da avaliação direta da distribuição de tamanho de partículas. Revista Engenharia Sanitaria e Ambiental 21(3), 1-8 (2016).

Moruzzi R. B.; Oliveira, A. L.; Conceição, F. T.; Gregory, J. and Campos, L. C. Fractal dimension of large aggregates under different flocculation conditions. Science of the Total Environment, 609, 807-814 (2017).

Moruzzi, R.B., Reali, M.A.P. Characterization of microbubble size distribution and flow configuration in DAF contact zone by a nonintrusive image analysis system and tracer tests, Water Sci. Technol. 61(1), 253-262 (2010).

Parker D. S., Kaufman W. J. and Jenkins D. Floc break-up in turbulent focculation processes. J. Sanit. Eng. Pro. Div. Am. Soc. Civ. Eng. 98 (SA1). 79-99. (1972).

Ringl, C. and M. Urbassek, H. A simple algorithm for constructing fractal aggregates with predetermined fractal dimension. Computer Physics Communications, 184, 1683-1685 (2013)

Santos, H. R.; Prado, G. S.; Vidal, C. M. S.; Moruzzi, R. B. \& Campos, J. R. Aplicabilidade das técnicas de determinação de tamanho de partículas em sistemas de tratamento de água e esgoto sanitário. Revista Brasileira de Engenharia Sanitária e Ambiental, 9(4), 291 - 300, (2004).

Smoluchowski, M. von. Versuch einer Mathematischen Theorie der Koagulations Kinetik Kolloid Losungen. Z. Physikalische Chemie., 92(155), 129-168 (1917).
Spicer, P.T., Pratsinis, S.E. Shear-induced flocculation: The evolution of floc structure and the shape of the size distribution at steady state. Water Research, 30, 1049-1056 (1996).

Tambo N. and Hozumi H. Physical Characteristics of Flocs-II.Strength of Floc. Water Research,.13, 421427. (1979).

Thomas, D.N.; Judda, S.J.; Faucett, N. Flocculation modelling: a review. Water Research, 33(7), 157992 (1999).

Vahedi, A., Gorczyca, B. Predicting the settling velocity of flocs formed in water treatment using multiple fractal dimensions, Water Research, 46, 4188-4194 (2012). http://dx.doi.org/10.1016/j. watres.2012.04.031

Verwey E. J. W.; Overbeek J. Th. G. Theory of the Stability of Lyophobic Colloids. Elsevier, Amsterdam. (1948).

Wei, J. Comments on paper: "Evolution of aggregate size and fractal dimension during Brownian coagulation" by Margaritis Kostoglou, Athanasios G. Konstandopoulos. Journal of Aerosol Science, 81, 21-23 (2015).

Wilkinson, N.; Metaxas, A.; Brichetto, E.; Wickramaratne, S.; Reineke, T. M. and Dutcher, C. $\mathrm{S}$. Ionic strength dependence of aggregate size and morphology on polymer-clay flocculation. Colloids and Surfaces A, 529, 037-1046 (2017).

$\mathrm{Wu}, \mathrm{J}$. He, C. Experimental and modeling investigation of sewage solids sedimentation based on particle size distribution and fractal dimension. Int. J. Environ. Sci. Tech., 7 (1), 37-46 (2010).

Yang, Z.; Yang, H.; Jiang, Z.; Huang, X.; Li, H.; Li, A.; Cheng, R. A new method for calculation of flocculation kinetics combining Smoluchowski model with fractal theory. Colloids and Surfaces A: Physicochem. Eng. Aspects 423, 11-19 (2013).

Yukselen, M.A., Gregory, J. The reversibility of floc breakage, Int. J. Mineral Process. 73, 251259 (2004). http://dx.doi.org/10.1016/S03017516(03)00077-2

Zhong, R.; Zhang, X., Xiao, F. and Li, X. Efects of humic acid on recoverability and fractal structure of alum-kaolin flocs. Journal of Environmental Sciences, 23 (5), 731-737 (2011).

Yu, W., Gregory, J., Campos, L., Graham, N., Dependence of floc properties on coagulant type, dosing mode and nature of particles. Water Research 68, 119-126 (2015).

Watanabe, Y., Flocculation and me, Water Research 114, 88-103 (2017), doi: 10.1016/j.watres.2016.12.035. 